\section{Late breaking news symposium}

\section{A1 PRIMARY RESULT OF THE SECOND MULTICENTRE INTRAPLEURAL SEPSIS (MIST2) TRIAL; RANDOMISED TRIAL OF INTRAPLEURAL TPA AND DNASE IN PLEURAL INFECTION}

${ }^{1} \mathrm{NM}$ Rahman, ${ }^{2} \mathrm{~N}$ Maskell, ${ }^{3} \mathrm{CWH}$ Davies, ${ }^{4} \mathrm{~A}$ West, ${ }^{5} \mathrm{R}$ Teoh, ${ }^{5} \mathrm{~A}$ Arnold, ${ }^{6} \mathrm{D}$ Peckham, ${ }^{7} \mathrm{~N}$ Ali, ${ }^{8} \mathrm{~A}$ Bentley, ${ }^{9} \mathrm{Cl}$ Mackinlay, ${ }^{10} \mathrm{WJ}$ Kinnear, ${ }^{1} \mathrm{JM}$ Wrightson, ${ }^{1} \mathrm{HE}$ Davies, ${ }^{11} \mathrm{RF}$ Miller, ${ }^{12} Y C G$ Lee, ${ }^{1} E L$ Hedley, ${ }^{1} \mathrm{~N}$ Crosthwaite, ${ }^{13} \mathrm{~L}$ Choo, ${ }^{13} \mathrm{~J}$ Darbyshire, 'FV Gleeson, ${ }^{13}$ AJ Nunn, 'RJO Davies. 'Oxford Centre for Respiratory Medicine, Oxford, UK; ${ }^{2}$ Southmead Hospital, Bristol, UK: ${ }^{3}$ Royal Berkshire Hospital, Reading, UK: ${ }^{4}$ Medway Maritime Hospital, Gillingham, UK; ${ }^{5}$ Castle Hill Hospital, Cottingham, UK; ${ }^{6}$ St James Hospital, Leeds, UK; ${ }^{7}$ Kingsmill Hospital, Mansfield, UK; ${ }^{8}$ Wythenshawe Hospital, Manchester, UK; ${ }^{9}$ Great Western Hospital, Swindon, UK; ${ }^{10}$ Oueen's Medical Centre, Nottingham, UK; ${ }^{11}$ University College London, London, UK; ${ }^{12}$ University of Western Australia, Perth, Australia; ${ }^{13}$ MRC Clinical Trials Unit, London, UK

\section{doi:10.1136/thx.2009.127035a}

Background Pleural infection affects $>65000$ patients per annum (UK + USA). $35 \%$ of these patients die or require surgery. Previous evidence suggests no benefit from intrapleural fibrinolytic agents although this is still debated. Direct plasminogen activators (tissue plasminogen activator (tPA)) and DNase to disrupt pleural biofilms and decrease pus viscosity may improve outcomes alone or in combination, but have not been tested in randomised trials.

Methods The Second Multicentre Intrapleural Sepsis (MIST2) trial is a double-blind, placebo-controlled $2 \times 2$ factorial trial of intrapleural tPA and DNase. 210 patients with microbiology-positive pleural fluid, or $\mathrm{pH}<7.2$ or purulent pleural fluid in the correct clinical context, from 11 centres were randomised to: doublematched placebo, active tPA plus active DNase, active tPA plus placebo DNase, or placebo tPA plus active DNase for 3 days (tPA $10 \mathrm{mg}$ bd, DNase $5 \mathrm{mg}$ bd). The primary outcome was change in chest radiograph effusion opacity from day 1 to day 7 postrandomisation. Group unblinded data only are presented here

Results Participants were 58.8 (SD 17.8) years, 60\% male; 173/199 $(87 \%)$ had community-acquired infection. Baseline characteristics were balanced across the treatment arms. Combination tPA/DNase was significantly superior to the other interventions. There was no evidence of improved drainage with tPA or DNase alone (absolute reduction in size of effusion as a percentage of hemithorax, tPA/ DNase 29.7\% SD 23.1; placebo/placebo 17.1\% SD 24.1; tPA/placebo 15.1\% SD 17.4; DNase/placebo 17.1\% SD 19.6; test for interaction $p=0.002,95 \%$ CI $5.0 \%$ to $22.9 \%$ ). The proportion of patients dying or requiring surgery for their infection was higher in the DNase/ placebo group and similar in all other groups (number of deaths or surgery, tPA/DNase 9/52 (17.3\%); placebo/placebo 7/55 (12.7\%); tPA/placebo 8/52 (15.4\%), DNase/placebo 23/51 (45.1\%), $\chi^{2}$ $3 \mathrm{df}=20.3, \mathrm{p}=0.0001)$. The occurrence of serious haemorrhage was not different between the groups (4 cases overall).

Conclusion Combination intrapleural tPA/DNase substantially improves pleural fluid drainage in pleural infection. DNase or tPA alone are ineffective and DNase alone appears to be associated with an increased frequency of surgery/death. The frequency of haemorrhage is not increased by these treatments. 